\title{
Reflets
}

Revue ontaroise d'intervention sociale et communautaire

\section{La formation à distance : un moyen novateur pour promouvoir la santé}

\section{Francine Deroche et Anne Simard}

Volume 5, numéro 2, automne 1999

La santé des francophones de l’Ontario

URI : https://id.erudit.org/iderudit/026279ar

DOI : https://doi.org/10.7202/026279ar

Aller au sommaire du numéro

Éditeur(s)

Reflets : Revue ontaroise d'intervention sociale et communautaire

ISSN

1203-4576 (imprimé)

1712-8498 (numérique)

Découvrir la revue

Citer cet article

Deroche, F. \& Simard, A. (1999). La formation à distance : un moyen novateur pour promouvoir la santé. Reflets, 5(2), 213-224.

https://doi.org/10.7202/026279ar
Résumé de l'article

Cet article trace les grandes lignes d'un projet de formation à distance qui a été mis en oeuvre en Ontario français. Plusieurs leçons ont été tirées de cette expérience qui peuvent servir dans la planification de futures activités de formation.

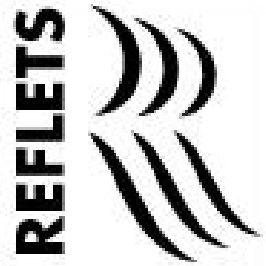




\section{La formation à distance : un moyen novateur pour promouvoir la santé}

F rancine $D$ eroche et $A$ nne Simard ${ }^{1}$

\section{Introduction}

Comment rejoindre les communautés francophones dispersées un peu partout dans la province et qui cherchent à se prendre en main, malgré un contexte politique et économique difficile? Par quelsmoyens peut- on leur fournir les outils dont elles ont besoin et ce, de façon efficace? Ce moyen existe. II est novateur et accessible, il s'agit de la formation à distance.

C et article trace les grandes lignes d'un projet de formation à distance qui a été mis en œuvre en 0 ntario français. Plusieurs leçons ont été tirées de cette expérience qui peuvent servir dans la planification de futures activités de formation.

\section{Besoins}

La communauté franco-ontarienne est défavorisée sur les plans de la langue et de la culture. Le Centre ontarien d'information en prévention (COIP) a reçu une subvention de Santé $C$ anada en 1997. Cette subvention a permis de mettre en œuvre un projet de formation à distance pour outiller davantage les francophones 
en 0 ntario afin qu'ils puissent composer avec des sujets del'heure, notamment I'action communautaire et les stratégies de financement.

$O \mathrm{n}$ avait constaté que les façons traditionnelles pour obtenir du financement et pour offrir les services aux communautés, ne fonctionnaient plus. Plus que jamais, les communautés francoontariennes devaient maximiser leurs ressources existantes et collaborer pour assurer leur survie.

La formation à distance se présentait comme un moyen novateur et accessible pour offrir cette formation et ainsi, rejoindre les gens à la grandeur de la province sans qu'ils aient à se déplacer et à encourir les dépenses rattachées au déplacement.

U ne étude de besoins, effectuée en $1997^{2}$, confirme que les intervenantes et les intervenants francophones en promotion de la santé ont une forte préférence pour la formation régionale et à distance.

\section{Buts et objectifs}

Ce projet de formation à distance visait à :

- mettre en œuvre une action communautaire efficace;

- assurer la durabilité de projets et d'activités communautaires;

- favoriser l'entraide entre divers groupes sur les plans régional et provincial;

- maximiser l'utilisation des ressources existantes en français en O ntario, et

- apprivoiser lesgensàl'utilisation destechnologiesinteractives.

\section{Mise en œuvre du projet}

L'équipe du projet, composée de consultantes travaillant pour le compte d'innovaction, a assuré la coordination, la planification, la 
mise en œuvre et l'évaluation du projet au nom du COIP. U n comité consultatif ${ }^{3}$ a été établi pour offrir ses conseils tout au long du projet. Les membres du comité consultatif ont apporté leur expertise à trois niveaux : leurs connaissances des réalités de leurs communautés, leurs expertises en matière d'action communautaire et de partenariats et leurs expertises en matière de prestation de formation.

\section{Conception pédagogique}

En tenant compte des besoins de formation, l'équipe du projet a assuré la conception pédagogique de deux séries d'ateliers ayant pour thème $D$ émystifions le communautaire et $D$ es stratégies de financement durables: pour soutenir vos projets et vos adivités et ce, en collaboration avec le comité consultatif et l'équipe d'animation.

Pour chaque série d'ateliers, des résultats d'apprentissage et des activités d'apprentissage ont été élaborés et organisés en modules. D eux guides ont été élaborés pour chaque thème: un guide de la participante et du participant et un guide pour l'équipe d'animation. Les guides incorporaient des ressources existantes en français.

$D$ ans l'atelier D émystifions le communautaire, les résultats d'apprentissage escomptés étaient que l'apprenante ou l'apprenant démontre, de façon suivie, sa capacité:

1. d'élaborer une stratégie d'action communautaire dans le but de répondre aux besoins de sa communauté, et

2. de mettre en pratique des stratégies qui facilitent le travail de groupe dans le but de répondre aux besoins de sa communauté.

En ce qui a trait à l'atelier $D$ es stratégies de financement durables: pour soutenir vos projets et vos activités, les résultats d'apprentissage étaient que l'apprenante ou l'apprenant démontre, de façon suivie, sa capacité:

1. d'élaborer une stratégie de financement dans le but d'obtenir les ressources nécessaires pour soutenir des initiatives communautaires; 
2. d'élaborer une proposition en vue d'obtenir les ressources nécessaires pour soutenir une initiative communautaire, et

3. d'évaluer l'apport des partenariats comme un moyen de soutenir des initiatives communautaires.

\section{Prestation}

Le R éseau franco-ontarien d'éducation à distance a coordonné les sites d'accès, les techniciens et les technologies interactives. Le $R$ éseau regroupe les sites d'accès de formation à distance des collèges francophones et des universités bilingues de l'O ntario. Lessites d'accès ciblés comprenaient C ornwall, Sudbury,T immins et $\mathrm{H}$ amilton.

Ce sont les animatrices et les animateurs régionaux, les expertes de contenu et les invités spéciaux, tous de l'O ntario français, qui ont assuré la prestation de la formation et ce, par le biais des technologies interactives et d'activités face-à-face se déroulant dans chaque site d'accès. Les animatrices et les animateurs régionaux et l'experte de contenu ont assuré le bon déroulement des sessions, facilité le travail de groupe et servi de ressources pour les apprenantes et les apprenants. Les invités spéciaux, utilisés seulement dans le cadre de l'atelier D es stratégies de financement durables, ont fait des interventions très ciblées de courte durée.

La formation a été offerte simultanément avec l'aide d'expertes de contenu, d'animatrices et d'animateurs régionaux et des technologies interactives disponibles dans deux sites d'accès: Sudbury et $C$ ornwall. La combinai son de technologies interactives et d'animation régionale cherchait à aider les apprenantes et les apprenants à développer leurs compétences en matière d'action communautaire et de financement, et aussi à faciliter leur apprentissage quant à l'utilisation des technologies interactives. D e plus, l'animation régionale permettait d'adapter l'approche afin de refléter les réalités régionales.

Pour chaque série d'ateliers, une audioconférence regroupant l'équipe d'animation se déroulait avant la session de formation afin de s'assurer que tous étaient sur la même longueur d'ondes. 
De plus, les animatrices et les animateurs régionaux et les expertes de contenu ont été formés par rapport à l'utilisation des technologies interactives.

\section{Mise en œuvre}

La série $D$ émystifions le communautaire s'est déroulée le $1^{\text {er }}$ et le 8 novembre 1997 à Cornwall et Sudbury. La deuxième série, D es stratégies de financement durables: pour soutenir vos projets et vos adivités, a été offerte le 29 novembre et le 6 décembre 1997 à C ornwall et à Sudbury.V ingt-six personnes ont participé à chacune des deux séries d'ateliers de formation. Bien qu'on ait prévu offrir les ateliers dans quatre villes, la formation a eu lieu seulement à Cornwall et Sudbury en raison du peu d'inscriptionsà $\mathrm{H}$ amilton et àT immins.

\section{Évaluation du projet}

\section{Résultats de l'évaluation lors de la formation}

La majorité des apprenantes et des apprenants ont rempli les formulaires d'évaluation. Toutefois, vu le petit nombre d'évaluations, il est seulement possible d'en faire une interprétation générale, de noter des tendances et d'identifier des pistes de réflexion plutôt qu'en tirer des conclusions fermes.

Les apprenantes et les apprenants semblent avoir apprécié les deux ateliers. Presque toutes et tous se sont dits satisfaits tant du contenu que du processus et des outils. Les animatrices et les animateurs régionaux, les expertes de contenu et les invités spéciaux connaissaient bien la matière et ont aidé à I'apprentissage. Les apprenantes et les apprenants ont bien aimé les activités en groupe, mais ont beaucoup moins apprécié les vidéoconférences. $L$ a réticence à la vidéoconférence, si marquée dansle premier module de la série $D$ émystifions le communautaire, s'est toutefois dissipée, témoignant sans doute du plus grand confort des apprenantes et des apprenants, des expertes de 
contenu et des animatrices et des animateurs régionaux. Les modes de prestation sembleraient s'apprendre autant que le contenu des ateliers.

\section{Résultats du suivi}

Bien que les apprenantes et les apprenants se soient dits satisfaits et ont dit avoir appris beaucoup lors de la formation, un suivi s'est avéré nécessaire afin de vérifier jusqu'à quel point les apprenantes et les apprenants ont atteint les objectifs de la formation et ont pu intégrer ces connaissances dans leur travail.

U ne seconde évaluation a donc été effectuée un an plus tard. II n'est pas surprenant que le taux de réponse de cette seconde évaluation soit nettement inférieur. En effet, seulement huit questionnaires ont été remis, reflétant l'expérience de quatre apprenantes et apprenants à $D$ émystifions le communautaire et de quatre égal ement, à D es stratégies de financement durables. Étant donné le petit nombre d'évaluations, il est toujours seulement possible d'en faire une interprétation générale, de noter des tendances et d'identifier des pistes de réflexion.

\section{Démystifions le communautaire}

Les apprenantes et les apprenants estiment que cette formation leur a permis d'accroître un peu mieux leur efficacité dansl'action communautaire. Tous disent qu'elle leur a fourni de nouveaux outils de travail tels le tableau pour un plan d'action, les noms de personnes-ressources et une meilleure connaissance du processus de prise de décisions. Leur capacité de mettre en pratique des stratégies qui facilitent le travail de groupe est nettement meilleure et leur capacité d'élaborer une stratégie d'action communautaire s'est améliorée.

\section{Des stratégies de financement durables}

Tous estiment que cette formation a accru leur capacité d'élaborer une stratégie de financement pour soutenir des initiatives et, une très forte majorité croit que leur capacité d'élaborer une proposition pour obtenir les ressources nécessaires aux initiatives 
communautaires s'est améliorée. Leur capacité de développer des partenariats pour soutenir les initiatives communautaires s'est accrue. La formation a permis à une majorité de développer des compétences supplémentaires telle la rédaction de propositions. Les deux tiers rapportent que la formation leur a fourni des nouveaux outils de travail.

Les trois quarts des apprenantes et des apprenants qui ont répondu à cette seconde évaluation estiment que la formation leur a été très utile dans le cadre de leurs différentes activités communautaires. Selon une majorité, la formation a favorisé l'entraide entre les divers groupes tant sur le plan régional qu'entre les villes de Sudbury et Cornwall. Presque tous croient que leur capacité d'utiliser les ressources humaines et matérielles existantes en 0 ntario français s'est améliorée.

La réaction des apprenantes et des apprenants demeure mixte quant aux technologies interactives. D eux disent que leur degré de confort s'est beaucoup amélioré grâce à la formation, trois disent qu'il s'est un peu amélioré et trois demeurent aussi confortables avec ces technologies qu'ils l'étaient avant de suivre la formation. Q uatre apprenantes et apprenants croient que I'utilisation destechnologies interactives a favorisél'apprentissage, tandis que deux maintiennent qu'elle a défavorisé l'apprentissage. Ces réponses ressemblent à celles obtenues lors de la première évaluation et suggèrent que, dans la critique de l'utilisation des technologies interactives, le problème porte davantage sur le mode de leur utilisation que d'un malaise inhérent à la technologie comme telle.

Les apprenantes et les apprenants estiment que le soutien qui leur a été offert était bon. La documentation écrite a été très bien reçue et presque tous la jugent très utile. La majorité de celles et de ceux qui ont répondu à la deuxième évaluation suivraient volontiers une autre formation. 


\section{Leçons}

II y a plusieurs leçonsqu'on peut tirer à partir de cette expérience, particulièrement en ce qui a trait au recrutement et à la prestation.

\section{Recrutement}

Le projet visait à l'origine quatre centres urbains: Cornwall, Sudbury, $\mathrm{H}$ amilton et $\mathrm{T}$ immins. II a été possible de rejoindre un nombre suffisant d'apprenantes et d'apprenants dans les deux premières villes, mais pas dans les deux dernières.

II y a quelques facteurs importants à considérer afin que la formation puisse être offerte dans plus de centres. II est essentiel que le comité consultatif comprenne des individus qui sont des têtes de réseaux dans les villes ciblées. Les membres du comité consultatif jouent un rôle très important en ce qui a trait à la promotion des activités de formation dans leur région. Sur notre comité, nous avions des représentants de Sudbury, Cornwall, $\mathrm{H}$ anover etWelland. $\mathrm{N}$ otre expérience avec Sudbury et Cornwall nous enseigne que la sollicitation personnelle des membres dans leur région a grandement incité les gens à participer. L'absence de représentants de $\mathrm{H}$ amilton et de $\mathrm{T}$ immins peut possiblement expliquer, du moins en partie, pourquoi nous ne sommes pas parvenus à attirer suffisamment d'apprenantes et d'apprenants dans ces villes. De plus, une représentation de chaque ville au sein du comité consultatif aurait pu permettre que la formation proposée réponde bel et bien aux besoins des apprenantes et des apprenants potentiels.

Compte tenu que les intervenantes et les intervenants et les bénévoles en région sont souvent surchargés, il peut s'avérer difficile pour eux de promouvoir efficacement les activités à titre de membre d'un comité consultatif. II serait donc valable de considérer la possibilité d'offrir des honoraires aux membres du comité consultatif pour qu'ils puissent y consacrer le temps nécessaire dans le cadre de leur boulot. 
Le matériel de promotion était bon, et l'on n'y attribue pas la responsabilité pour les difficultés de recrutement. La promotion a été faite par un envoi postal ciblé d'un dépliant promotionnel, suivi d'un encart invitant à obtenir de plus amples renseignements. Plus précisément, on a ciblé les quatre villes et leurs alentours. U n communiqué de presse a également été envoyé aux médias. O $n$ a aussi utilisé d'autres réseaux pour faire la promotion des atelierscomme lescoordonnateurs des Services de santé en français et les centres de santé communautaire francophones, etc. En plus d'allonger la période de temps pour le recrutement, on pourrait explorer de nouveaux moyens comme l'insertion d'un dépliant ou placer des annonces très brèves dans les journaux, etc.

Ceci étant dit, notre expérience nous dit que le recrutement se fait beaucoup de bouche à oreille et par l'entremise de réseaux informels en région, d'où l'importance capitale du comité consultatif.

\section{Prestation}

En dépit des difficultés de recrutement, nous sommes satisfaits du nombre de personnes inscrites. $N$ ous recommandons toutefois que lors d'une prochaine formation, le nombre d'apprenantes et d'apprenantspar région soit limité à un maximum de 15 personnes et ce, pour faciliter l'apprentissage tant au niveau du contenu que des technologies interactives. La leçon principale à retenir d'une formation à distance, c'est que la pédagogie doit mener la barque et non pas les technologies interactives. Les technologies interactives doivent servir et s'adapter aux apprenantes et aux apprenants et à l'équipe d'animation.

La grande majorité des apprenantes et apprenants avaient les compétences nécessaires pour suivre la formation, bien que certains aient dû se familiariser avec plusieurs nouveaux concepts, surtout dans l'atelier D émystifions le communautaire. De plus, il y avait une série importante d'apprentissage à faire concernant l'utilisation des technologies interactives. II serait donc préférable de commencer la formation, en région, avec une activité face-à-face qui permette aux gens de faire une entrée en matière à partir de 
ce qu'ils connaissent et aussi, de leur donner le temps d'apprivoiser lestechnologiesavant d'être obligés de lesutiliser en temps virtuel.

Les besoins d'apprentissage de la clientèle ont été bien identifiés, et le contenu de la formation était bien adapté à ces besoins. N ous sommes satisfaits des guides d'apprentissage destinés aux apprenantes et aux apprenants et des expertes de contenu. Les guides offrent une occasion d'auto-apprentissage, allant audelà de ce qui était couvert dans la formation et suggérant des pistes de recherche personnelles à explorer. Les animatrices et animateurs régionaux et les expertes de contenu connaissaient très bien leur matière et les guides ne sont là que pour donner certaines balises. 0 utre les guides, les animatrices et les animateurs régionaux et les experts de contenu ont été bien encadrés et soutenus avant et pendant la formation. Soulignons l'importance de former d'avance les animatrices et les animateurs régionaux et les expertes de contenu afin qu'ils soient à l'aise avec la technologie employée au cours de la formation.

U ne variété de moyens d'apprentissage a été employée au cours de la formation à l'aide des technologies interactives et d'activités régionales de face à face. C ette approche a bien fonctionné dans son ensemble. Elle a permis une formation semblable à Sudbury et à C ornwall, tout en respectant les besoins particuliers des régions et en offrant des occasions d'échange de face-à-face. C estechnologies ont facilitéle dialogue entre régions et ont suscité l'entraide entre les groupes.

C hose certaine, les apprenantes et les apprenants apprécient I'accès à divers outils et la participation à des activités $d$ 'apprentissage qui permettent de mettre en pratique leurs connaissances. L'utilisation d'une variété d'activités d'apprentissage, individuel ou de groupe, est également à recommander. La combinaison des guides, de face-à-face en région et l'échange inter-régional est fort enrichissant.

Le face-à-face est un élément indispensable. À cet égard, les animatrices et animateurs régionaux ont fait une contribution tout à fait essentielle. D ansun contexte où la souplesse est limitée par la tenue de la formation dans deux sites simultanément, les 
animatrices et animateurs régionaux offrent un soutien important aux apprenantes et aux apprenants un peu moins expérimentés. Leur approche personnelle a permis de faire le pont entre la théorie et la pratique, de passer des concepts au concret et d'étoffer la formation de vraies histoires. Ils ont également pu faciliter l'apprentissage face aux technologies interactives et refléter les réalités régionales dans la formation.

Les apprenantes et les apprenants ont fait preuve d'une ouverture aux technologies interactives, mais leur degré de confort avec la vidéoconférence dépendait de l'experte de contenu et de son degré de maîtrise de cette technologie. $\mathrm{N}$ ous suggérons que d'autres types de technologies soient intégrés à la formation, possiblement par Internet ou par l'usage du CD-ROM, ce qui rendrait la formation encore davantage accessible.

\section{Conclusion}

Pour lescommunautésfrancophones dispersées à traversl'0 ntario, la formation à distance représente un moyen efficace pour les outiller. Toutefois, la formation à distance a ses limites et il faut trouver des moyens pour surmonter les défis qu'elle présente. U ne prestation qui met à profit à la fois lestechnologies interactives et l'animation régionale semble être une formule prometteuse à cet égard.

M algré le succès remporté, il y a encore beaucoup de pain sur la planche afin que la formation à distance fonctionne efficacement et que toutes les communautés aient accès aux outils dont ils ont besoin pour faire leur travail.

Somme toute, nous sommes satisfaits de la formation et nous répéterions volontiersl'expérience. $N$ ousavonsatteint les objectifs esquissés, soit de développer les compétences en action communautaire et en stratégies de financement, de faciliter l'échange entre régions, de maximiser l'utilisation des ressources existantes et d'apprivoiser les gens aux technologies interactives. 
N ous lançons le défi à nos homologues de considérer une semblable démarche pour desservir les communautés dispersées avec lesquelles ils travaillent ${ }^{4}$.

\section{Notes}

1. Les auteures sont consultantes pour la firme innovaction, 180, rue $D$ undas 0 uest, bureau 1900,Toronto (O ntario) M 5G 1Z8, _http:// www.innovaction.com, courrier électronique: francine@ innovaction.com ou anne@ innovaction.com

2. Le Centre ontarien d'information en prévention et le $\mathrm{C}$ entre for $\mathrm{H}$ ealth Promotion de l'université deToronto (1997). É tude de besoins en matière des services de langue française en promotion de la santé

3. Lesmembres du comité consultatif étaient :Jocelyne B lais-B reton, Services en français de l'hôpital Welland County General, M arc Bisson, Centre de santé communautaire de l'Estrie, Josée Lamarche, Les Entreprises Josée F. Lamarche, et Louise Picard, Service de santé publique de Sudbury et du district.

4. Certaines ressources élaborées dans le cadre de ce projet sont disponibles au site web suivant : http:/ / www.innovaction.com. II s'agit d'un bulletin qui résume les grandes lignes de la formation et qui identifie des ressources supplémentaires de deux feuillets qui font la synthèse des renseignements fournis dans le cadre de la formation, et d'une version adaptée du guide de I'apprenante et l'apprenant D es stratégies de financement durables 